\title{
Tailored Fiber Placement in Thermoplastic Composites
}

\author{
A. Spickenheuer ${ }^{1)}$, C. Scheffler ${ }^{1)}$, L. Bittrich ${ }^{1)}$, R. Haase ${ }^{2)}$, D. Weise ${ }^{2)}$, D. Garray ${ }^{3)}$, G. Heinrich ${ }^{1)}$, \\ 1) Leibniz-Institut für Polymerforschung Dresden e.V., spickenheuer@ipfdd.de, Hohe Straße 6, \\ 01069, Dresden, Germany \\ 2) Fraunhofer Institute for Machine Tools and Forming Technology IWU, Chemnitz, Germany \\ 3) SIRRIS, Liège/Wallonia, Belgium
}

\section{Keywords}

Fiber placement, thermoplastic, commingled yarns, sizing, PBT, processing, incremental sheet forming

\begin{abstract}
Fiber path optimization methods combined with the Tailor Fiber Placement (TFP) technology provide the optimum correlation between load case and fiber orientation and therefore lead to unmatched component performance with endless fiber composite materials. The aim of this work is the development of an innovative manufacturing technology for thermoplastic composites (TPC) including sizing-adapted commingled glass fiber (GF) / thermoplastic yarns (SpinCom yarns) to be processed by TFP to textile preforms with a variable-axial, load adapted fiber design. Furthermore, these preforms will be consolidated in a low energy and resource consuming process using novel light and low cost forming tools produced by incremental sheet metal forming technology. Finally, a low cost solution for thermal processing even for complex shaped TPC parts will be presented. Heading towards optimized resource and cost efficiency of the whole process chain, first results of SpinCom yarns, fiber path optimization, tool manufacturing and forming procedure are presented and demonstrated using GF/PBT (polybutylene terephthalate) SpinCom yarns and the geometry of a bicycle saddle.
\end{abstract}

\section{Introduction}

The development of fiber-reinforced plastics (FRP) opens up a high strength, stiffness and lightweight potential for structural components. To utilize the full performance capabilities, the orientation of the anisotropic fibers has to match the specific load case. However, in most cases layered structures of unidirectional (UD) yarns, so called multiaxial laminates, are used for composite manufacturing. This is mostly motivated by the availability of textile semi-finished products, e.g. woven and non-woven fabrics, and well-established numerical methods for a multiaxial laminate design. However, this results in significantly reduced component performances. Fiber path optimization methods combined with Tailored Fiber Placement can provide the optimum correlation between load cases and fiber orientation and therefore leads to unmatched component performance with composite materials. For simple shapes and plain loads new methods for the design and modeling of variable-axial composite structures made by TFP have been developed recently [1], [2], [3], [4]. If SpinCom yarn is applied by the TFP technology, the resulting preforms are further processed by hot pressing using mostly plane molds [5]. However, especially topological optimized truss-like variable-axial TFP preforms are very well suited for a three-dimensional draping of complex shaped composite parts. Nonetheless, even the manufacturing of such consolidations molds is highly complex and is bound to high material losses. Additionally, the so far applied thermoset resin infusion process does not result in constant high quality composite properties of the final FRP part. On the example of the lightweight stool L1, the required tool made of four parts, the milling process of the mold with its high material losses and the resin rich zones of the vacuum bagged composite parts are shown in Figure 1 [6]. In order to minimize 
manufacturing costs and efforts and to improve the overall laminate consolidation quality of complex shaped thermoplastic composites at once, the cooperation of different scientific fields is necessary. To achieve this objective, a new type of thermoplastic hybrid yarn, new methods for 3D modeling of variable-axial composites, a novel way to manufacture consolidation molds as well as the enhancement of the consolidation process for TPC based preforms are under development.
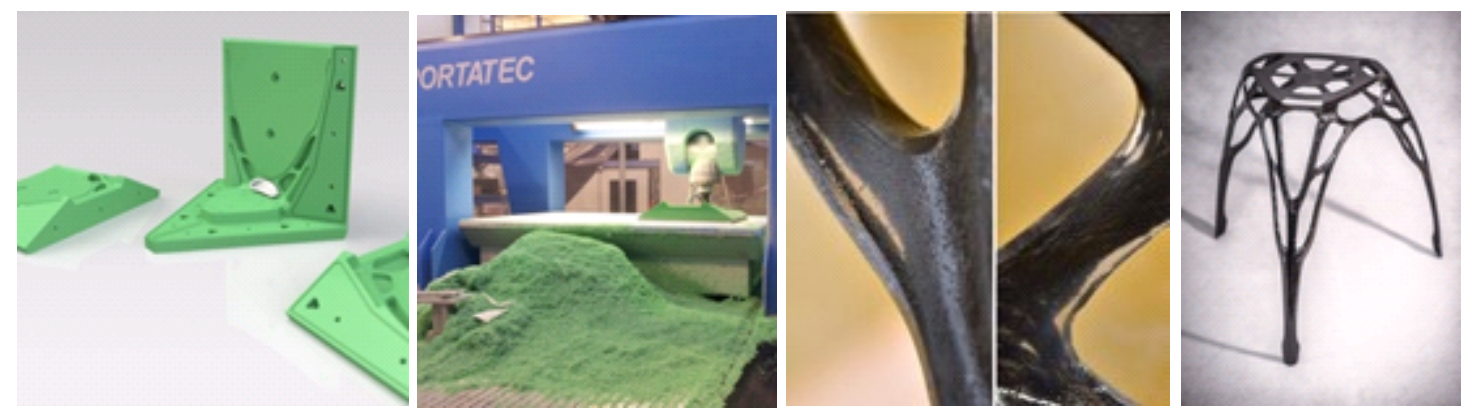

Figure 1: Lightweight stool L1 - mold concept for thermoset infusion, mold manufacturing by milling, close up of resin agglomerations (transparent areas), final lightweight stool L1 (from left to right) [6]

\subsection{Commingled yarns}

The application goal of working with high performance thermoplastic composites is fully in accordance with the capacity of IPF to produce online commingled yarns in a simultaneously spinning process [7]. The impregnation quality in endless fiber reinforced thermoplastic composite is often insufficient because of the high viscosity of thermoplastic matrices. During the desired short cycle times in the consolidation process, the molten thermoplastic matrix is not able to penetrate the small spaces between the several thousand single reinforcing filaments. The basic idea to solve this problem is the deposition of the thermoplastic material between the filaments already before the consolidation process as it is realized in commingled yarns. However, existing products on the market reveal some weak points: the hybrid yarns produced by airjet texturing reveal damaged reinforcing fibers due to the swirl process; still, it allows combining nearly every kind of reinforcing and thermoplastic filament. Also the side-by-side structures of hybrid yarns enable very different material combinations but show deficits in the homogenous distribution. Additionally, previously applied spin finishes on the thermoplastic filaments might reduce the wetting behavior or the fiber-matrix adhesion. One of the most promising routines is provided by SpinCom yarns, which are based on the simultaneous spinning of reinforcing (GF) and thermoplastic filaments that are commingled during the spinning process to avoid further process steps and achieve more homogeneous filament distributions. In previous tests, different matrix polymers (PP, PLA, PA) and sizings have been investigated by the processing of SpinCom yarns with the aim to improve the interfacial adhesion of their composites [9], [10]. In this work, GF/PBT SpinCom yarns are chosen as an optimal material for preforming by TFP to produce high quality complex structural lightweight parts.

\subsection{Tailored Fiber Placement}

The TFP technology offers high quality in preform manufacturing of highly curvilinear placed reinforcing fibers at a high productive level. For this reason it is still highly competitive compared to new technologies like endless fiber reinforced 3D printing or Automated Fiber Placement (AFP). The main principle of this technology is well described by [8] and shown in Figure 2. 

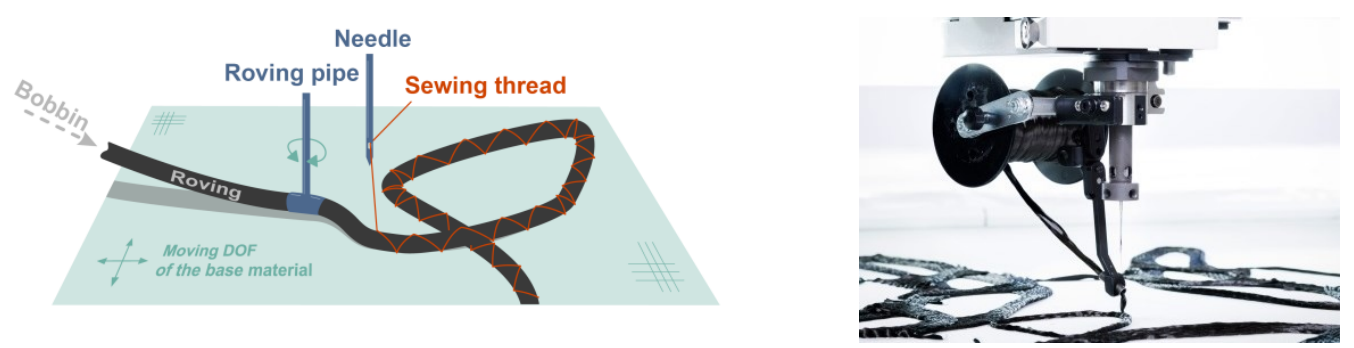

Figure 2: Principle of the Tailored Fiber Placement (left) and detail of the TFP processing of a carbon yarn (right)

The current handicap to utilize the TFP technology in a much more extensive way is due to the missing of applicable modeling and optimization software tools for curvilinear designed composite parts. First approaches to model variable-axial composites made by TFP are still limited to two dimensions [2]. Further, it is still unclear what is the best way to design a complex three-dimensional curved variable-axial TFP part. In general, two options are possible: 1) Design and modifying the fiber paths in 3D and finally unfold the fiber pattern into 2D to obtain an TFP trajectory pattern or 2) optimize an inherently manufacturable 2D fiber pattern and project it to the final 3D shaped composite part model for further numerical investigation.

\subsection{Incremental sheet forming (ISF)}

The opportunity proposed by the ISF technology to produce light and low cost tooling for small series [11] also targets economic advantages for the whole process. Additional to the pure lightweight impact, especially the reduced thermal capacity and therefore accelerated temperature increase and low energy consumption for the tempered forming process is aimed. Moreover, ISF offers short timeto-market capabilities and is cost effective for small and medium lot sizes as well as a high degree of geometrical flexibility, Figure 3.
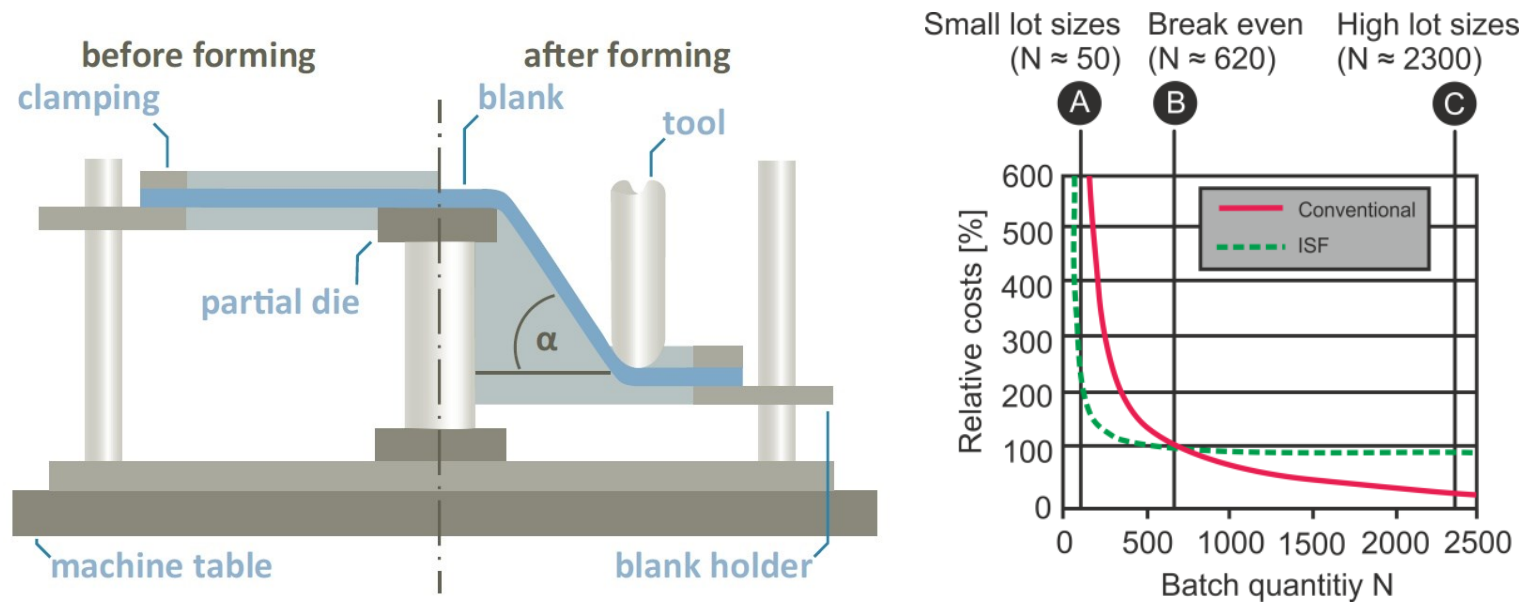

Figure 3: Principle of increment sheet forming (left), final ISF part (middle) and application numbers vs. costs per unit [12]

For the intended production of a consolidation mold, the Single-Point-ISF (without supporting grid) or for tools with higher geometric complexity the ISF with partial die (grid) support will be utilized [13], [14].

\subsection{Thermoplastic composite consolidation}

The optimum processing technology in terms of structural performance for 3D TPC products is given by the usage of a hot pressing process. However, those closed mold processes are very cost intensive in case of mold manufacturing and the final part shape is limited to due to necessary draft 
surfaces. In order to consolidate complex shaped TPC parts, the usage of a vacuum bag processing supported by a single sided mold and an autoclave with high temperature cycle capacity up to $400{ }^{\circ} \mathrm{C}$ can be a feasible approach. Figure 4 illustrates a demonstration part (3D vane made of carbon/PA12 fabric preform) produced using this technique, proving the feasibility of the method.
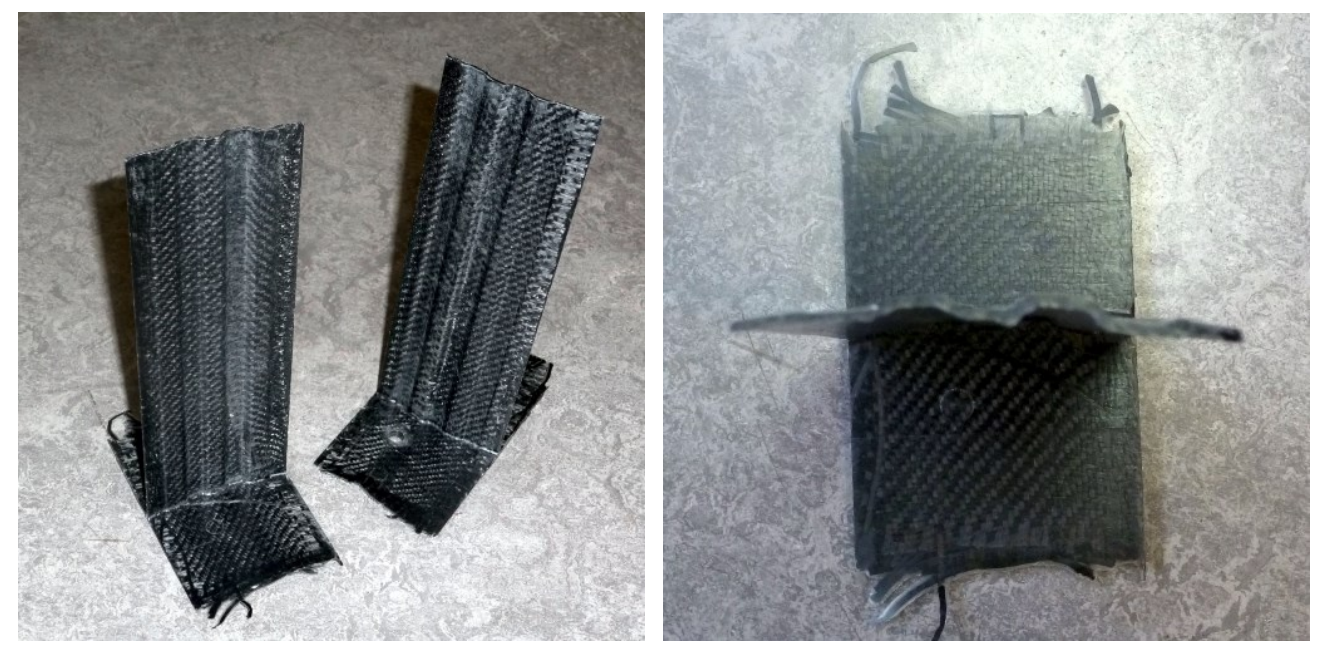

Figure 4: 3D CF/PA12 vane produced by using autoclave technology

Autoclave processing is known as a high-end technique and allows the production of optimum complex 3D parts, but this technology is not economic in terms of a broad industrial application. Consequently, alternative solutions such as vacuum bag systems at standard pressure could be used to replace the costly, high-end process. A core target of this alternative process route is to increase the economic performance while the mechanical properties of the product remain outstanding.

\section{Hybrid yarn processing}

\subsection{Manufacturing of GF/PBT-SpinCom yarns}

The commingled yarns are made by simultaneous spinning of E-glass fibers (GF) and Polybutylenterephthalat (PBT) filaments with different sizings. Two kinds of PBT filaments are produced with different melt volume rates (MVR) out of raw materials from two commercial suppliers: PBT1 with MVR $=46 \mathrm{~cm}^{3} / 10 \mathrm{~min}$ and PBT2 with MVR $=19 \mathrm{~cm}^{3} / 10 \mathrm{~min}\left(250{ }^{\circ} \mathrm{C} ; 2.16 \mathrm{~kg}\right)$. Different sizing formulations have been compared during the spinning process (Table 1). The sizings have been applied by the sizing roll, which also commingles the two filament types. In this way, both filaments, GF and PBT, are in contact with the same sizing. The optimized processing conditions for a homogeneous mix of the GF and PBT filament arrays has been established after comparing different technological routes including the adaption of the spinning velocity and the use of applicable nozzles for the PBT spinning. The melt temperatures of GF and PBT are $1200{ }^{\circ} \mathrm{C}$ and $260{ }^{\circ} \mathrm{C}$, respectively. The cooling behavior of the filaments controlled by the processing speed is crucial to determine the fiber diameter as well as the commingling and the winding process. PBT and glass filaments were spun at a speed of approximately $700 \mathrm{~m} / \mathrm{min}$ with fiber volume fraction of $50 \%$ and a fineness of about 160 tex. The cross sections of GF filaments show diameters of $16 \mu \mathrm{m}$ and PBT filaments of $25 \mu \mathrm{m}$ according to these processing conditions.

\subsection{Composite manufacturing and transverse tensile test}

Unidirectional PBT/GF composites were produced by filament winding (IWT Wickeltechnik, Erlangen, Germany) of the SpinCom yarns followed by compression molding. After filament winding, the mandrels were transferred to a computer (PC)-controlled platen press (KV207, Rucks, Germany) for the consolidation process. The parameters for composite manufacturing were defined by the isothermal temperature of $250{ }^{\circ} \mathrm{C}$ (PBT1) and $265^{\circ} \mathrm{C}$ (PBT2), respectively, a pressure of 2.17 bar over 
a time of $16 \mathrm{~min}$. The fiber volume fraction of the composites ranged from $49.7 \%$ (V6-3/PBT2) to $56.8 \%$ (V6-5/PBT2). Samples for the transverse tensile test were cut out of the composites and tested in accordance to DIN EN ISO 527-5.

Table 1: Sizing formulations applied during SpinCom yarn processing

\begin{tabular}{lll}
\hline Sizing & Sizing formulation & Matrix polymer \\
\hline V1 & $\begin{array}{l}\text { 3-glycidoxypropyltrimethoxysilane, } \\
\text { phenoxy film former } \\
\text { 3-aminopropyltriethoxysilane } \\
\text { epoxy film former 2 }\end{array}$ & PBT1 \\
V4 & $\begin{array}{l}\text { 3-glycidoxypropyltrimethoxysilane, } \\
\text { epoxy film former 3 (low content) }\end{array}$ & PBT1/PBT2 \\
V6-3 & $\begin{array}{l}\text { 3-glycidoxypropyltrimethoxysilane, } \\
\text { V6-5 }\end{array}$ & PBT1/PBT2 \\
unsized 1 & $\begin{array}{l}\text { none film former 3 (high content) } \\
\text { unsized 2 }\end{array}$ & PBT1 \\
\hline
\end{tabular}

\subsection{Results and Discussion}

After spinning and drying the SpinCom yarn bobbins, the behavior during the textile processing is highly dependent on the sizing, since well-running yarns are the precondition for further processing in TFP. Figure 5 shows that sizing V1 leads to damage and yarn splicing during winding. In contrast, yarns with sizing V4/PBT2 reveal good textile processability. Besides the bobbin quality, PBT2 was also found to lead to a higher level of the mechanical properties in the transverse tensile test (Figure 5). Also the results of the composites made of SpinCom yarns without sizing contribute to the good processability of PBT2: no significant difference in the strength was found, however, the standard deviation is strongly reduced. In both PBT polymers, composites with the sizing formulation V4 achieved the highest transverse tensile strength indicating a high fiber-matrix-interaction for the used film former/silane combination.

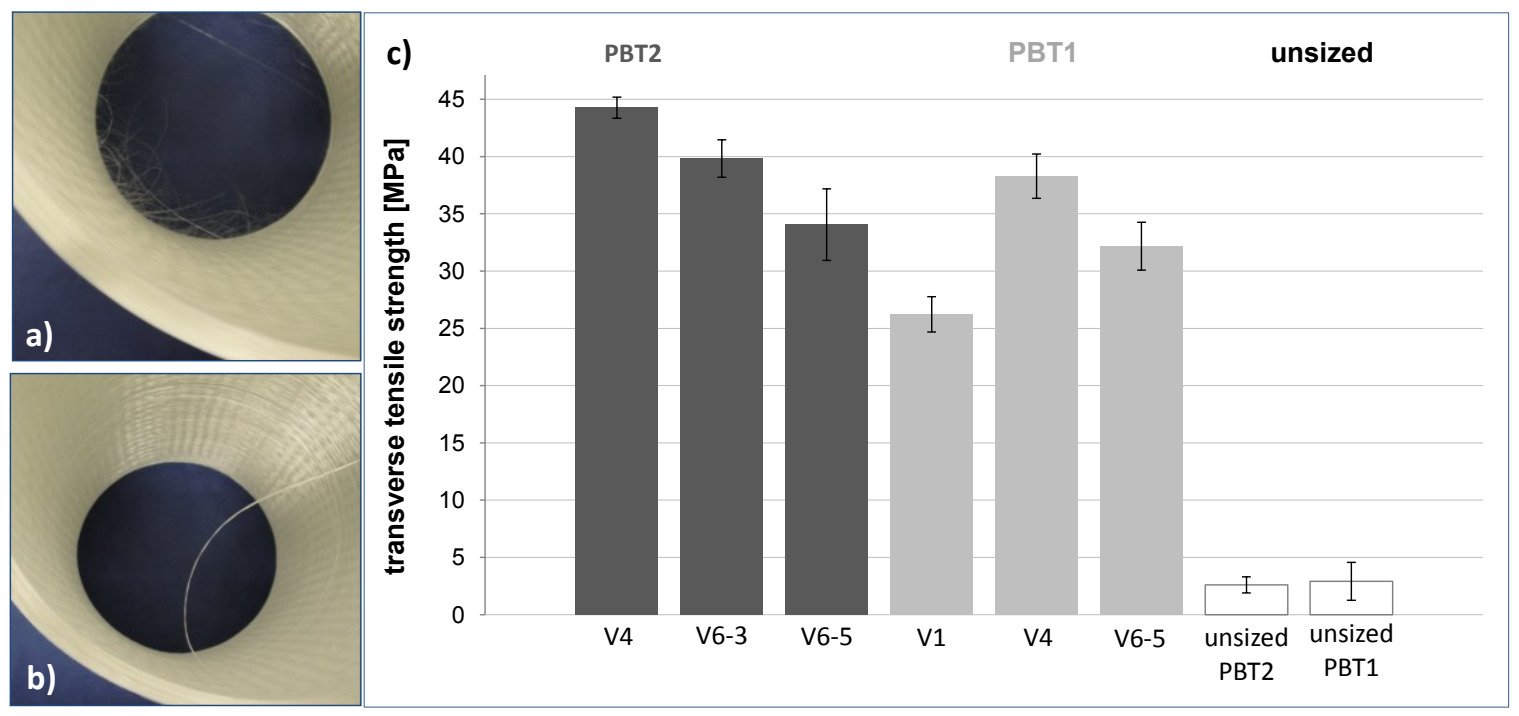

Figure 5: Comparison of SpinCom yarn fuzz/friction properties depending on the sizing: a) high fuzz formation (sizing V1), b) no fuzz (sizing V4) and c) transverse tensile strength of unidirectional PBT/GF composites depending on sizing formulation and type of PBT polymer 


\section{Optimization of 3D shaped variable-axial composites made by TFP}

\subsection{Optimization approach and modeling}

The current state of art to design a complex variable-axial TFP preform with help of numerical methods and software tools is limited to flat, two-dimensional (2D) structures. With the Direct Fiber Path Optimization (DFPO) method an optimization method was developed to modify 2D preform patterns by adjusting the limited number of spline control points only. After this realignment of fiber trajectories, the TFP placement pattern is mapped to an even 2D FE mesh considering the local preform thickness and fiber orientation in a shell- or solid element based finite element model [15]. In order to expand this modeling or rather optimization approach to complex variable-axial threedimensional shaped TFP composite structures, the numerical and practical draping procedures emanating from the 2D manufactured preform towards the real 3D part geometry is crucial. Draping of TFP preforms is severely limited so only small intrinsic curvatures are considered. For instance, the amount of applied sewing thread is limiting the potential shear deformation by impeding the reinforcing yarn locally. The deployment of standard draping software tools which generate cuts on highly shear deformed areas would be counterproductive in case of the application of fiber placement process like TFP. Instead, a draping model is used for small curvatures based on a minimization of deformation strains. A mapping for this draping is derived which connects flat 2D coordinates to the 3D shape and vice versa. This process enables an adaptation of the chosen modeling approach to unravel a meshing of the 3D shape to a flat surface. Based on an either manually or numerically generated TFP pattern, a subsequently generated 3D curved realistic FE model can be created automatically. Such a 3D shaped FE model also features all physically relevant local thickness and fiber orientation variations. Besides the opportunity to modify the in-plane fiber pattern manually, the automatic virtual draping procedure will be further integrated into the in-plane conducted DFPO optimization process.

\subsection{Example}

To demonstrate the enhancement of the modeling approach from any given $2 \mathrm{D}$ designed variableaxial TFP pattern into a 3D complex shaped CFRP part, a geometry possessing a double-curved shape was chosen. Such a geometrical characteristic could be found on a bicycle saddle, which was subsequently used for further numerical and experimental studies. In a first step, a real saddle of a racing bike was 3D scanned and transferred into a 3D CAD model as shown in Fig 6 (left). Based on the 3D shaped saddle geometry, the flattening was conducted. In Figure 6 (middle) the maximum required strain can be seen and in Figure 6 (right) the final flat geometry which implies the final contour for the plane TFP pattern design.
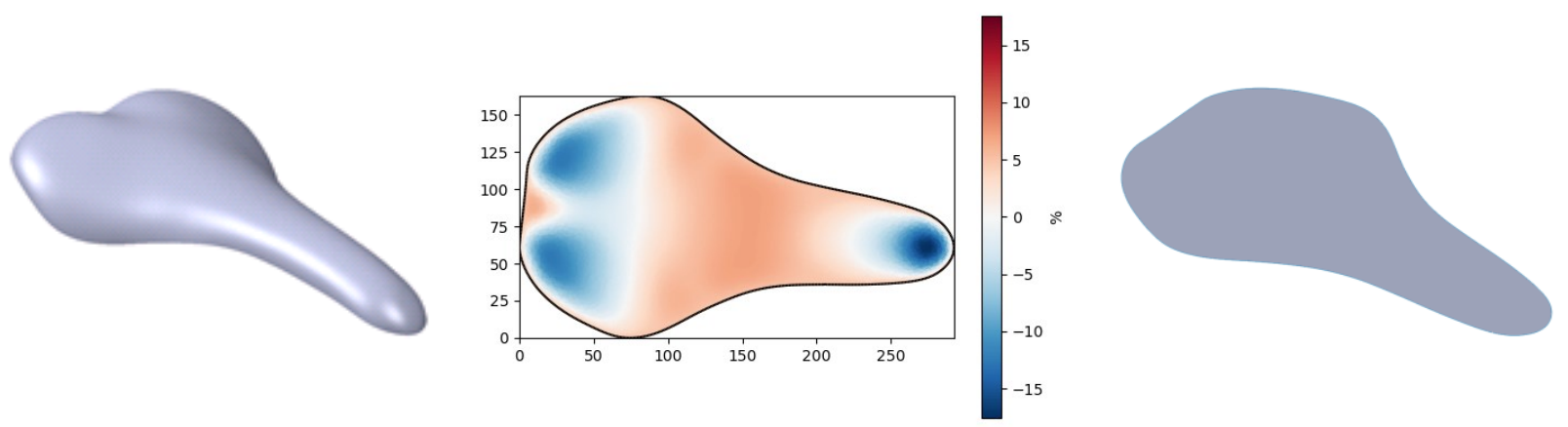

Figure 6: 3D-scan of a bicycle saddle (left), unfolded saddle geometry with indicated shear deformation areas (middle) and final flat saddle geometry (right)

The generic pattern design based on 2 layers of mostly vertical lines was semi automatically generated and is shown in Figure 7 (left). The same design pattern was also used to manufacture the final TFP preforms shown in Figure 12 (left). The corresponding 3D FE mesh of the saddle geometry is shown in Figure 7 (right). This FE mesh was used as template for the subsequent mapped meshing process. 

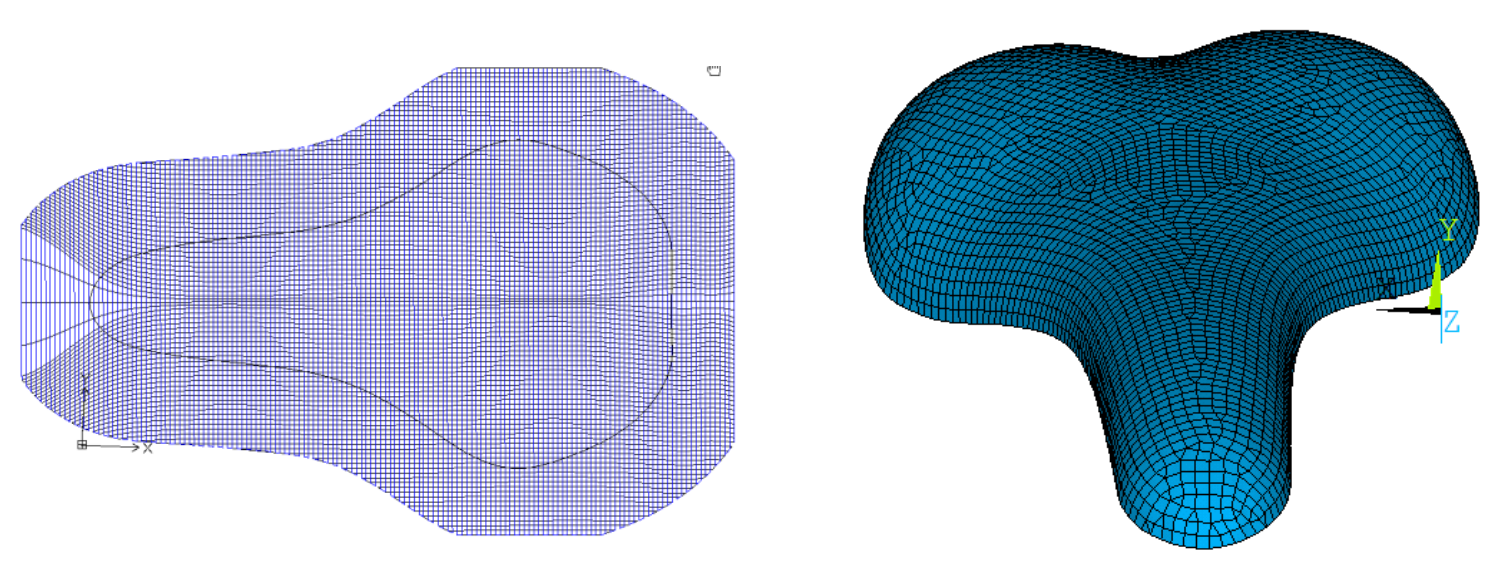

Figure 7: Fiber pattern design (left), 3D FE mesh of saddle (right)

Once the virtual transformation of the applied geometry is known, the same process can be repeated forward and backward also for FE meshes. With the already established mapping procedure of a local evaluation of solid element node thicknesses and element coordinate system orientations, as shown in Figure 8, the resulting 3D FE mesh can be generated [1].

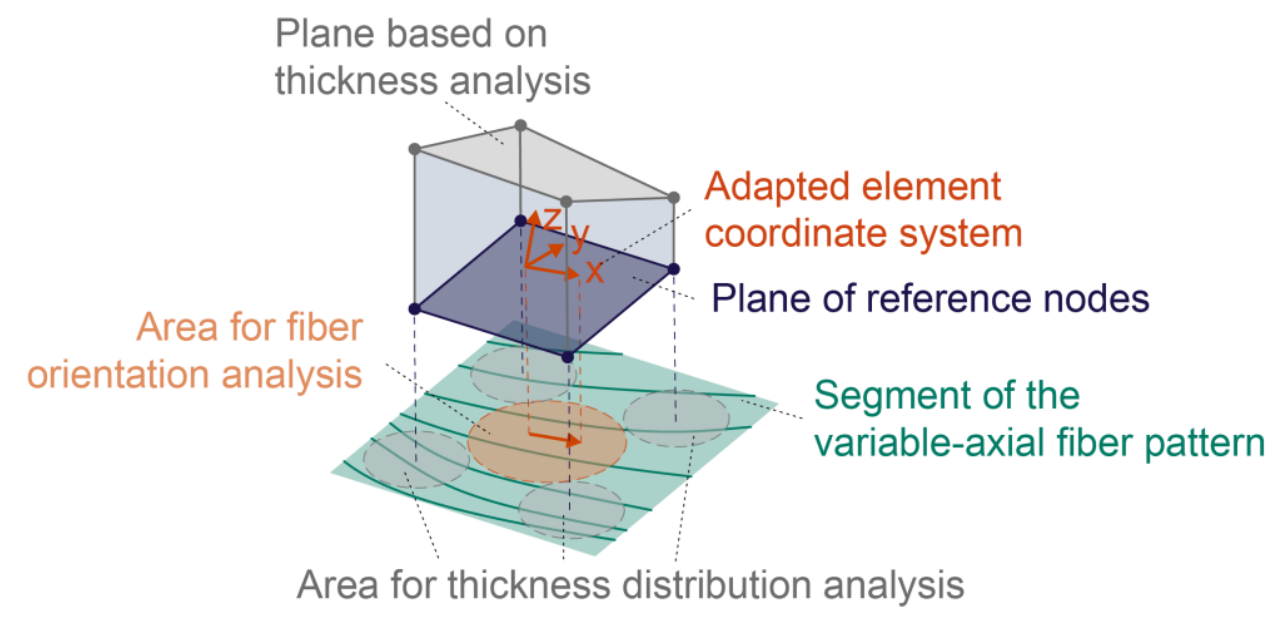

Figure 8: Modeling approach for deriving the finite solid element parameters,

e.g. thickness at corner nodes and element coordinate system alignment

The resulting FE mesh including the local element coordinate orientation of the two given TFP layers as shown in Figure 7 (left) can be seen in Figure 9. 

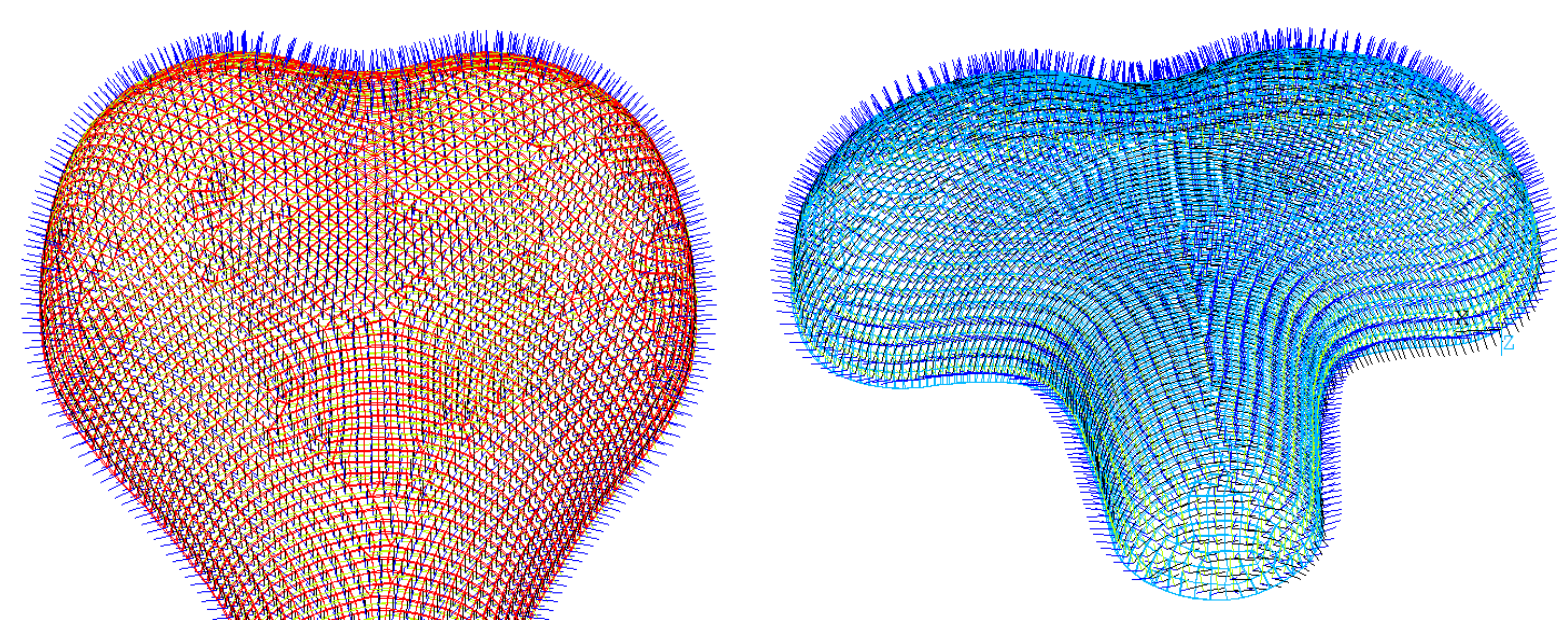

Figure 9: 3D FE mesh with derived element coordinate orientation for the first (left) and second (right) TFP layer applied

\subsection{Results and Discussion}

In contrast to optimization approaches, which work on the 3D shapes themselves, the presented approach only considers valid or rather manufacturable TFP patterns and the draping is defined at the beginning of the whole design process. For stronger curvatures a more realistic draping, which incorporates the anisotropic behavior of the TFP preforms as well as the sewing thread, should be considered. However, such an approach cannot be integrated in the DFPO procedure at this stage as the flat design space changes with each change in pattern.

For the given complex or rather double-curved geometry of the bicycle saddle, the developed modeling approach has been proven valid. Within the ongoing work, it will be applied to the DFPO optimization approach and finally demonstrated on further demonstration parts, e.g. the lightweight stool L1 shown in Figure 1.

\section{TPC consolidation molds made by incremental sheet metal forming}

\subsection{Manufacturing approach}

The aim of the incremental sheet forming (ISF) process is to generate a lightweight consolidation mold. In contrast to the state of the art, where the mold is generated by the milling of an initially solid block, the process already starts with a thin sheet of metal. The shape of the intended mold is formed by a sequence of kinematic movements of a trivial forming element. Thus, the geometry is not defined by the shape of the tool itself but by the kinematic sequence of the tooling along the forming path.

\subsection{Example}

Within the project presented in this paper, there are two stages of technology development and demonstration parts. In a first step, a shell-like component is regarded. Dedicated to the individual ergonomic needs, a bike saddle was selected as demonstrator. An initial CAD data set was achieved by an optic 3D scan of a commercial saddle. Subsequently, the point cloud was used for a re-design of the surface by definition of various cross sections and a connecting longitudinal spline. By combination of the sections, a curvature constant part model was generated and is available for further processing, including biaxial scaling and NC-programming.

Due to the use of a bike saddle, the functional surface is convex and therefore the corresponding mold face needs to be concave. To avoid surface defects on the contact side of the mold, the shell was formed from the backside, which is convex again. This layout implies a partial support die. To ensure a minimum resource impact, this die was milled from a wooden block, as shown in Figure 10. 

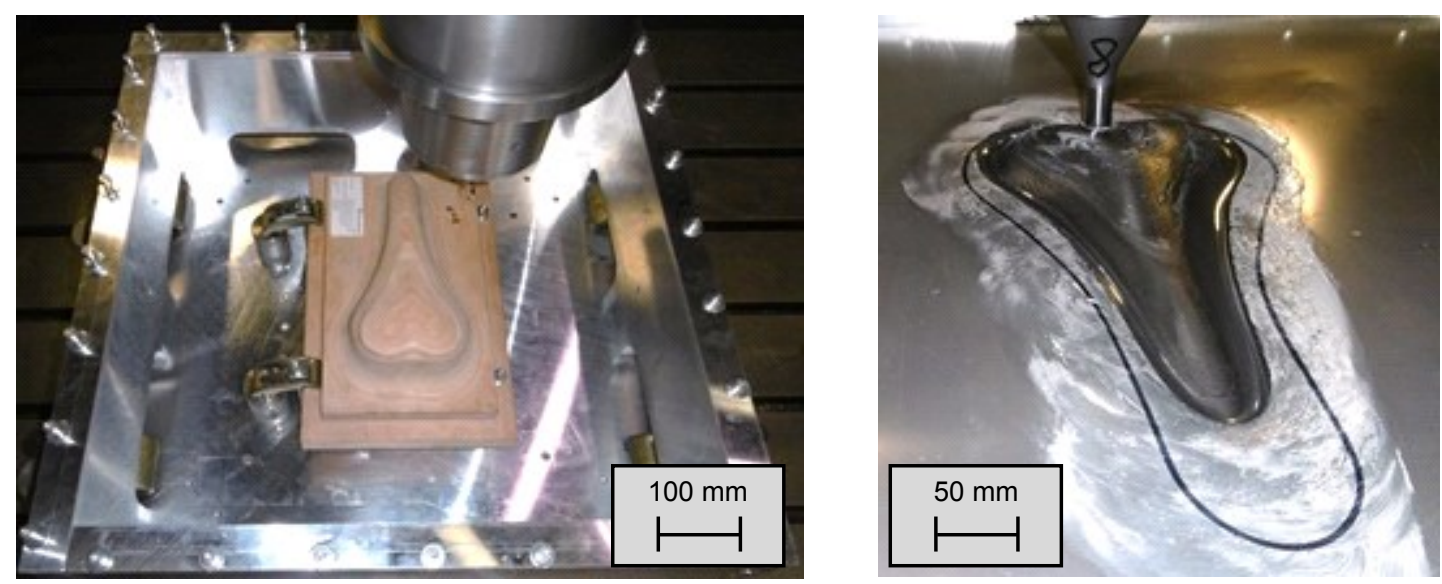

Figure 10: Machining setup for incremental sheet forming (ISF) including clamping frame, wooden partial die and CNC-axis moving the forming tool (left) and forming of narrow groove (right)

The initial forming regime was purely based on the sliced CAD geometry and generated a sharp groove around the saddle geometry. The repeated elongation of the material within the narrow band resulted in excessive thinning and finally appearing material failure even before the whole depth could be achieved. As a countermeasure, the narrow groove was replaced by a wider band and thus distributed the elongation in an extended area. The different geometric representations are shown in Figure 11.
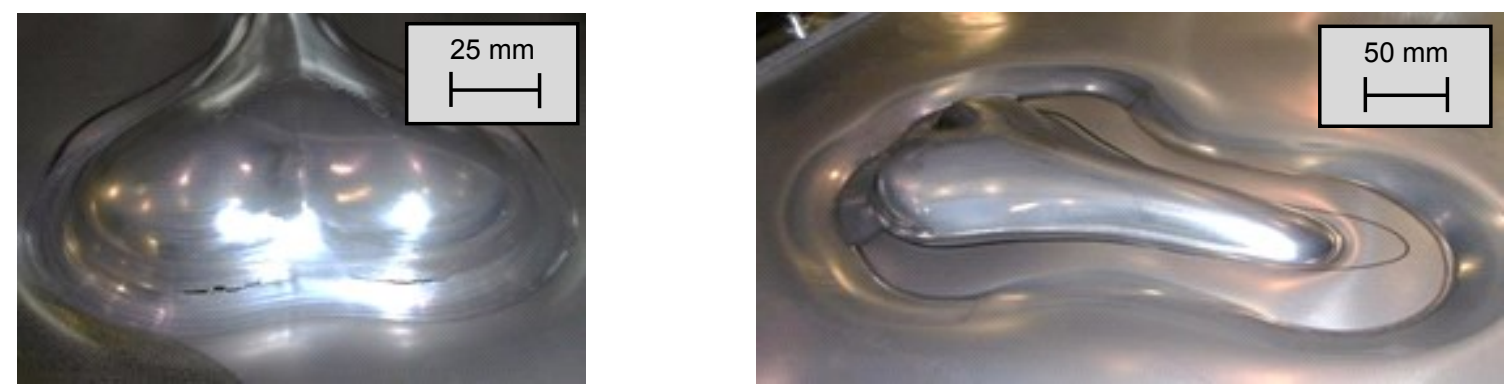

Figure 11: Material due to excessive thinning in narrow groove area (left) and countermeasure by increased forming area and thus reduced material thinning (right)

\subsection{Results and Discussion}

The elaboration of different ISF forming regimes and corresponding CNC-programmed paths of the forming tool did illustrate the impact of the process design on material thinning and feasibility of the intended computer aided design (CAD) geometry. On the final component, nearly perpendicular wall angles could be achieved. Different flange lengths were generated by a trimming process. This should enable a complete vacuum bagging solution (short flange) or a one-sided approach by application of a sealing on the elongated flange as an alternative. During the trimming operation, no distortion or twist of the shell could be observed. Due to the double-curved surface, the shell does provide a good stiffness, stability and shape integrity.

In a further project stage, the more complex geometry of the lightweight stool L1 will require a segmentation of the consolidation mold (ref. to Figure 1). By the symmetric design of the lightweight stool, the support die will contain only one segment of the full crown. A major objective will be the forming of a flange area by means of ISF within an integrated process. With regards to the increased complexity of the formed parts, a thermal support of the forming process can provide an extended forming limit as well as reduced deflection due to spring-back effects [16]. 


\section{Vacuum bag based consolidation of hybrid yarn based preforms}

\subsection{Methods and technology}

Conventional use of an autoclave (or an oven) to process composite parts is mostly limited to temperatures up to $200{ }^{\circ} \mathrm{C}$ that are large enough for a majority of thermoset (TS) materials (like epoxy based elements). The new generation of thermoplastic (TP) based continuous reinforced composites easily overcome this limit. Only polymers like PLA, polyolefins or some polyamides can be processed under this thermal barrier or slightly just above.

The autoclave available at SIRRIS allows processing temperatures up to $400{ }^{\circ} \mathrm{C}$, and so is fully compatible with the use of more technical polymers such as PBT, PC, PPS or even PEEK. Although the equipment can reach this target, it is not fully the case for some consumables and accessories that are needed to perform the consolidation of the TP preforms.

As an example, a polyimide membrane is proposed to replace the standard polyamide based vacuum bag. It has been proved that this solution is fully compatible to higher temperatures, but the lack of the flexibility of the membrane also required to adapt the way of working, especially by limiting the sharpness of some components of the tooling. Another product that causes some problems in finding an alternative solution is the vacuum channel that avoids the collapsing of the membrane and then connects the composite part to the vacuum valve during the whole processing cycle. The best and most economical solution we have found up to now is a standard steel wool. Finally, the sealing tape also requires some protocol adaptation because if the product is well known and available, it is also necessary to be coupled with a second tape that ensures the tightness at the lower temperatures.

Concerning the TFP technology, it has to be assumed that it is starting from a $2 \mathrm{D}$ architecture of a unique yarn to be developed during the consolidation phase in a 3D structure. So the use of an extra support is required for the handling and the cohesion of the yarn into the layer definition. Because the support is closely linked to the reinforcement structure, it will remain present in the final part. Then it is necessary to use a material that is strong enough to support the TFP preform during its creation, to be manipulated between the different processing units. It also needs to be flexible enough to follow the 3D shape without causing extra complications in terms of wrinkle creation. Finally, the material needs to be compatible with the matrix of the TP composite. Currently proposed solutions are a glass woven fabric, a glass fleece and a PA based film. In Figure 12, the pictures show the first samples available for consolidation trials (here with the PA based film) as well as the saddle tooling.
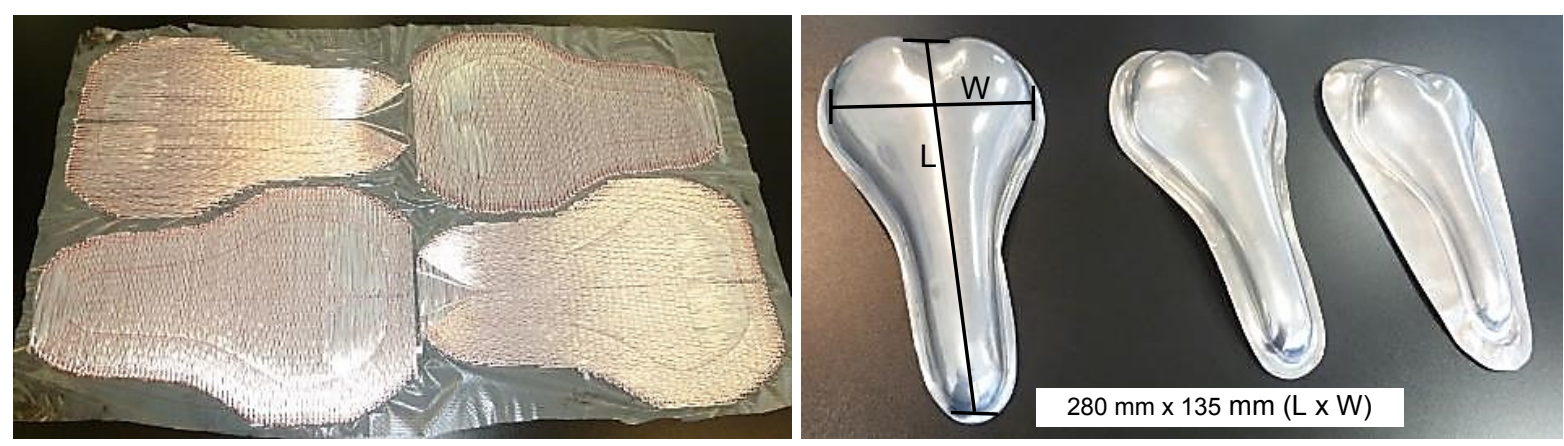

Figure 12: TFP-GF/PBT preforms with a PA base material (left) and saddle consolidation tools made by ISF (right)

\subsection{Consolidation tests on unidirectional specimens}

The first consolidation tests have been performed with the aim to characterize the material developed by the partner IPF using a combination of PBT and glass fibers. The other target of the approach was to test the new products (consumables, accessories ...) required for higher processing temperatures and finally to check the effect of some process parameters. In Figure 13 the equipment and the processing phase are shown. 

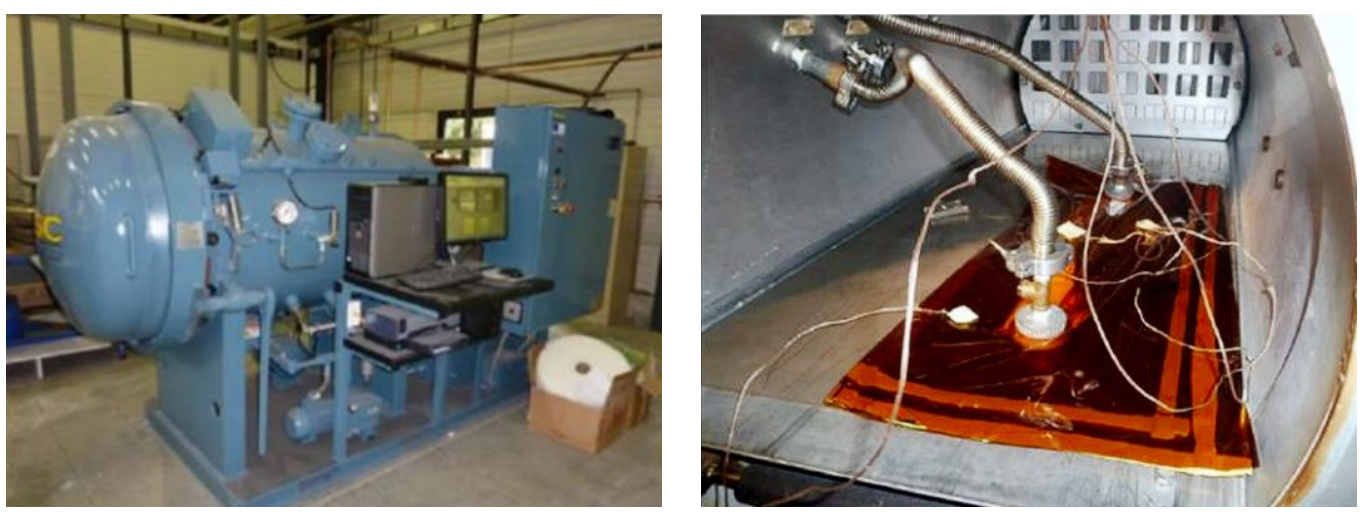

Figure 13: Autoclave (left) and process set-up for TFP-TPC consolidation (right)

The first results of the via autoclave technology consolidated TBC saddle made of TFP preforms and GF/PBT2 SpinCom yarn is shown in Figure 14 together with its corresponding ISF made mold. For the consolidation process, a temperature of $235^{\circ} \mathrm{C}$ and an additional pressure of 2 bar were applied. The red net-shaped surface results from a red colored commercial PBT stitching yarn, which was applied at the TFP process for roving fixation.

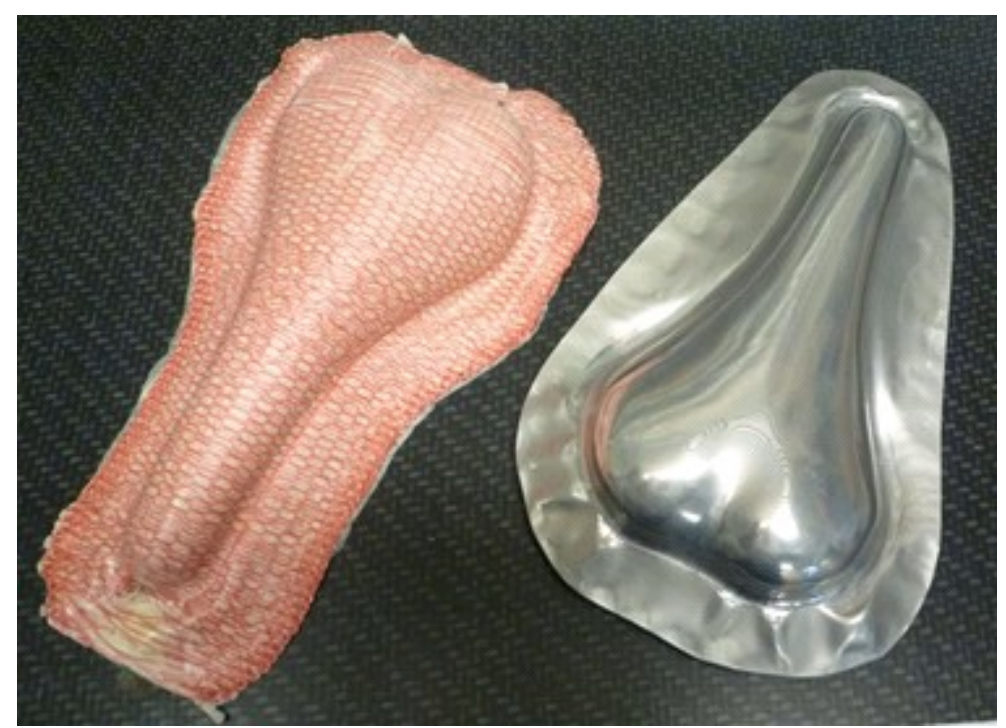

Figure 14: Consolidated GF/PBT composite part and consolidation mold made by incremental sheet forming

Because an objective of the project is to validate the capacity of a vacuum processing into an oven to consolidate TFP preforms, meaning without extra pressure like within an autoclave, specific trials have been performed in this way.

In Figure 15, the results of the tensile and bending tests are presented. They summarize the influence of the temperature $\left(235^{\circ} \mathrm{C}, 255^{\circ} \mathrm{C}\right)$ and of the pressure level ( 0 bar, $5 \mathrm{bar}, 10 \mathrm{bar}$ ) on the mechanical performances of PBT/GF yarns consolidated in a unidirectional architecture. All tests have been conducted within a standard atmospheric environment of $50 \%$ relative humidity and a temperature of $23^{\circ} \mathrm{C}$. For both, the tensile test according to ISO 527-2 as well as for the bending test according to DIN EN ISO 178, a standard testing machine (Instron 4507) was applied. Due to the small amount of hybrid yarns, produced mainly for preliminary studies, each mechanical test was carried out with only four specimens. This comparable low number of control samples is the reason, why the error bars in the diagrams of Figure 15 represent only the measured minimum and maximum values and do not show the standard deviation data, as would be usual. 


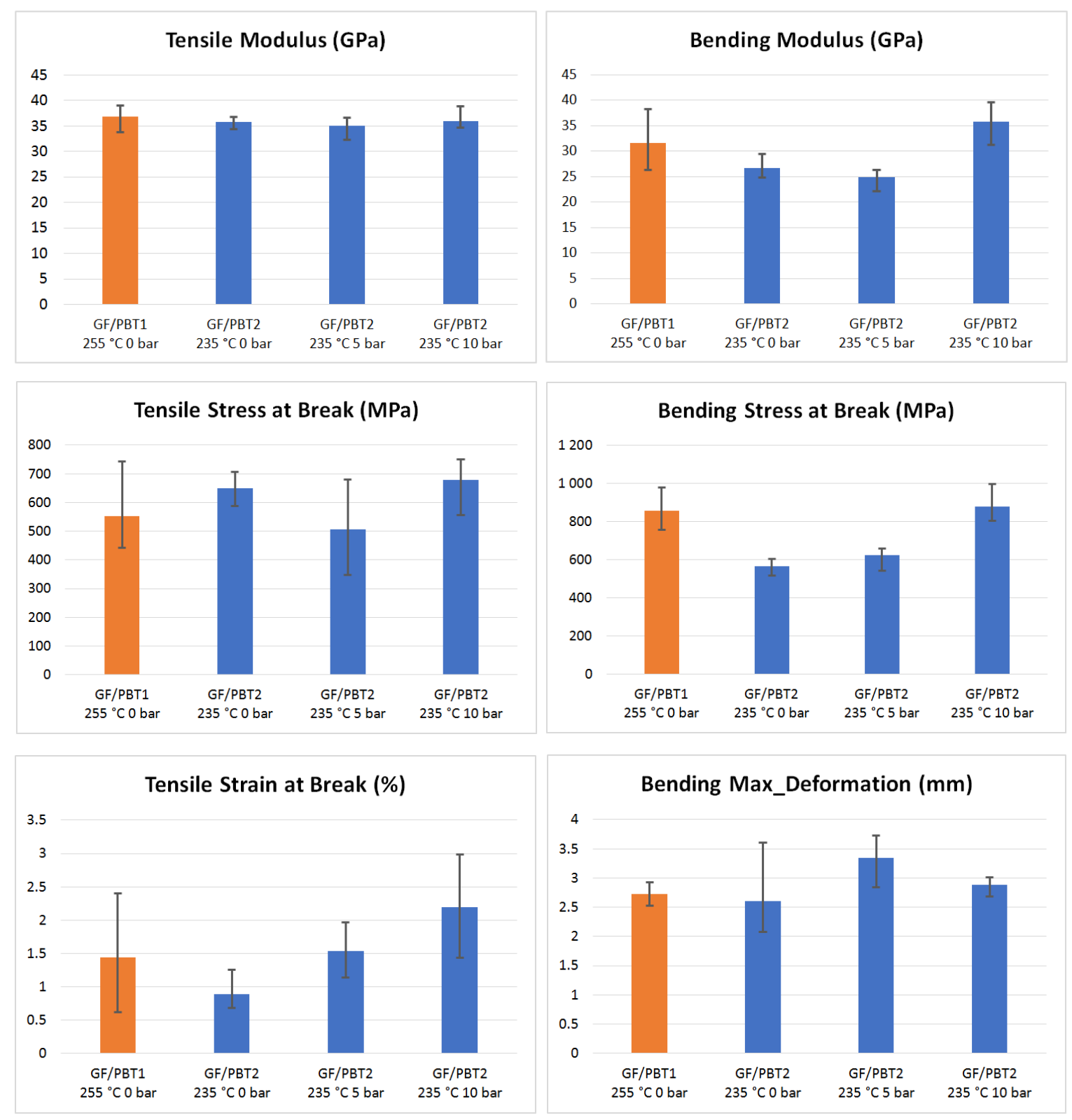

Figure 15: Experimental results of different PBT types and consolidation conditions, results tensile tests (left) bending test (right), the different colored columns represent the two different applied PBT types

\subsection{Results and Discussion}

The consolidation process of complex 3D shaped TFP preforms made by GF/PBT hybrid yarn material in ISF molds by using autoclave technology was demonstrated successfully. A first demonstration part was manufactured. However, process costs and manufacturing efforts need to be reduced further. Therefore, an out-of-autoclave TPC consolidation process is targeted. The experimental tests of TPC specimens manufactured with and without autoclave technology offer already promising outcomes.

The results of the tensile tests show that the effect of the extra pressure applied during the autoclave process is not significant except for the strain at break, which seems in direct link with the process parameter. The influence of the temperature seems also negligible. This situation can be explained by the nature of the tensile test that is mainly driven by the reinforcement fibers.

The bending characterization, on the other hand, is more representative by nature of the quality of the polymer phase and of the interface between the matrix and the glass fibers. By analyzing the bending modulus and stress at break as a function of the same parameters, it is clearly visible that the temperature, as well as the pressure, has a positive effect onto those properties. But, in link with our 
project objective, it also shows as positive result that the increase of temperature can compensate the lack of pressure in an out-of-autoclave process. In a next step, the achieved knowledge of out-ofautoclave TPC processing will be applied for the manufacturing of 3D-TFP composite structures.

\section{Conclusion and outlook}

With this work, we present novel strategies to design and produce composite structures with superior performance for lightweight applications. The combination of sizing-adapted SpinCom comingled yarn with the Tailored Fiber Placement technology allows high quality thermoplastic composites with precision placement and efficient material usage for preform production. Even though the modeling and optimization of such structures was limited to flat shapes prior to this work, the draping could be incorporated in the modeling of arbitrary TFP patterns. Thus, a general optimization of complex structures becomes possible. Furthermore, we demonstrated the applicability of incremental sheet metal forming to generate complex 3D shaped tools for thermoplastic composite consolidation. At the early stage of the presented project, the consolidation process was carried out with help of a high temperature autoclave. This facilitates the usage of high temperature matrix systems such as PBT, PC, PPS or even PEEK.

Future work will focus on broadening the range of applications by providing examples for segmented tools with even more flexibility of shapes. A fully autonomous optimization of the fiber placement pattern for such an example will allow a load adaptation of such a design with structural performance values at the limit of what is possible with these materials. The incremental sheet forming process will be applied to even more complex shaped tool geometries, which consist of several sectional sub molds. Finally, the TPC consolidation process will be developed towards an out-of-autoclave manufacturing. Therefore, vacuum bag technology with heated and easy to handle molds will be investigated in order to further increase cost-efficiency and energy saving.

\section{References}

[1] Spickenheuer, A.: Zur fertigungsgerechten Auslegung von Faser-Kunststoff-Verbundbauteilen für den extremen Leichtbau auf Basis des variabelaxialen Fadenablageverfahrens Tailored Fiber Placement, Disseration, Technische Universität Dresden, Fakultät Maschinenwesen, 2014.

[2] Spickenheuer, A.; Bittrich, L.; Uhlig, K.; Richter, E.; Heinrich, G.: Recent developments in simulation and processing of composite structures made by tailored fiber placement. In: 14th Japan International SAMPE Symposium and Exhibition (JISSE14), Ishikawa, Kanazawa, 06.12.2015 - 09.12.2015.

[3] Uhlig, K.; Spickenheuer, A.; Bittrich, L.; Heinrich, G.: Development of a highly stressed bladed rotor made of a cfrp using the tailored fiber placement technology, Mech. of Compos. Mater. 49 (2) (2013), pp. 201-210.

[4] Uhlig, K.; Tosch, M.; Bittrich, L.; Leipprand, A.; Dey, S.; Spickenheuer, A.; Heinrich, G.: Mesoscaled finite element analysis of fiber reinforced plastics made by tailored fiber placement, Compos. Struct., 143 (2016), pp. 53-62. doi: 10.1016/j.compstruct.2016.01.049

[5] Richter, E.; Uhlig, K.; Spickenheuer, A.; Bittrich, L.; Mäder, E.; Heinrich, G.: Thermoplastic composite parts based on online spun commingled hybrid with continuous curvilinear fibre patterns. In: ECCM 16 - 16th European Conference on Composite Materials. - Sevilla, Spanien, 22.06.2014 - 27.06.2014.

[6] Richter, E.; Spickenheuer, A.; Bittrich, L.; Uhlig, K.; Heinrich, G.: Applications of variable-axial fibre designs in lightweight fibre reinforced polymers. Mater. Sci. Forum 825-826 (2015), pp. 757-762. doi: 10.4028/www.scientific.net/MSF.825-826.757

[7] Mäder, E.; Rausch, J.; Schmidt, N.: Commingled yarns-Processing aspects and tailored surfaces of polypropylene/glass composites. Compos. Part A, 39 (2008), pp. 612-623. doi: 10.1016/j.compositesa.2007.07.011

[8] Mattheij, P.; Gliesche, K.; Feltin, D.: Tailored fiber placement - mechanical properties and applications, J. of Reinf. Plast. and Compos., 17-9 (1998), pp. 774-786.

doi: 10.1177/073168449801700901 
[9] Wiegand, N.; Mäder, E.: Commingled yarn spinning for thermoplastic/glass fiber composites. Fibers, 5 (2017), ID26. doi: 10.3390/fib5030026

[10] Wiegand, N.; Mäder, E.: SpinCom yarns - From reinforcement to functional fibres. In: Proceedings of the European Conference On Composite Materials 2014, ECCM-16, 2014.

[11] Weise, D.; Müller, R.; Demmler, M.; Scholz, P.: Efficient manufacturing technology for production of sheet metal parts for small and medium quantities. In: Conference on Advanced Metal Forming Processes in Automotive Industry (AutoMetForm), Freiberg, 2014, pp. 264-268.

[12] Hirt, G.: Inkrementelle Blechumformung als wirtschaftliche Alternative zur Herstellung von Prototypen und Kleinserien. In: 19. Aachener Stahlkolloquium, Umformtechnik - Stahl und NEWerkstoffe "Visionen und Innovative Lösungen", Mainz, Aachen, 25.-26.03.2004, S. 251-261.

[13] Jeswiet, J.: Rapid prototyping of a headlight with sheet metal. In: Transaction of NAMR/SME. Vol. XXX, 2002, pp. 33-38.

[14] Leach, D.: A new incremental sheet forming process for small batch and prototype parts. In: Proceedings of the 9th Int. Conference on Sheet Metal, SHEMET 2001, Leuven, pp. 211-218.

[15] Bittrich, L.; Spickenheuer, A.; Heinrich, G.: Composite design with variable-axial fibers by direct fiber path optimization. In: Congress on Numerical Methods in Engineering CMN2017, Valencia, 3-5 July 2017.

[16] Landgrebe, D.; Weise, D.; Reuther, F.; Meinel, S.; Scheffler, C.; Scholz, P.;Duflou, J.; Vanhove, H.; Mohammadi, A.; Auwers, W.; Troy, S.: Heat supported incremental sheet metal forming of lightweight materials. In: EFB Project Report 405, European Research Association for Sheet Metal Working, 2015, Hanover. 